\title{
Wave - particle duality interpretation: de brogile equation criticisms
}

\begin{abstract}
The development of the quantum theory has been on - going for many years. The wave - particle duality has been and is central to debates among physicists, in their endeavor to understand and bring more insight about the quantum theory. In this paper attempts by physicists to clarify the wave - particle duality are discussed. The wave or particle or both state of matter is analyzed, followed by discussion on the criticisms on de Broglie equation. Lastly, the paper concludes that debates on the wave - particle duality will continue since there is no consensus yet among physicists, and that experimental evidence should be given to substantiate claims.
\end{abstract}

Keywords: quantum theory, wave - particle duality, de brogile equation
Volume 4 Issue 6 - 2020

Alois Matorevhu
Department of Science and Mathematics, Mutare Teachers'
College, Zimbabwe

Correspondence: Alois Matorevhu, Department of Science and Mathematics, Mutare Teachers' College, 81 Chimanimani Road, Zimbabwe, Tel +263773936 455,

Email amatorevhu@gmail.com

Received: November 25, 2020 | Published: December 15, 2020

\section{Introduction}

In the historical development of quantum theory, wave - particle duality has been one of the guiding concepts in intensive debates on the quantum theory. Variations in interpretations of the wave - duality result in different meanings, hence lack of consensus among physicists. This paper sought to analyse the variations in interpreting the wave - particle duality. To achieve this, various theoretical arguments, experimental results and their interpretations were analysed. It was found that based on one's philosophical position the purpose of interpreting the wave - particle duality could be predicting or explaining phenomena or making a reality claim in a hidden structure or the process of nature. This analysis revealed that particles and waves are mutually exclusive in classical physics, since they exhibit different behaviours. In quantum mechanics, as postulated by de Broglie both the particle and wave behaviours are embedded in matter, but they cannot be simultaneously exhibited. While de Broglie is applauded for a break through which provided knowledge for guiding further development of quantum mechanics, criticisms which need attention have been highlighted in this paper. It is concluded from the analysis that the complexity of interpretation of the wave - particle duality is a fact, which is an indication that debates related to interpretation of the wave - particle duality, and specifically de Broglie equation, will not end in a foreseeable future. Therefore in order to bring more insight, physicists should continue debating these issues as well as putting effort to provide experimental evidence, to substantiate claims.

\section{Attempts to clarify wave - particle duality}

In the historical development of quantum theory, wave - particle duality has been one of the guiding concepts. ${ }^{1}$ While intensive debates in relation to the interpretation of the quantum theory are on - going, it is important to note that duality was one of the key issues in the early debates concerning the quantum theory. Debate over the wave - particle duality concerning visible light which started in the times of Isaac Newton when modern physics was being born, was radically changed by quantum mechanics. The ambiguity of the structure of matter is exactly as that of light, such that the plausible way out of the wave or particle stalemate is to take a dualistic stance, implying light and matter sometimes behave like particles, and sometimes like waves, depending on (experimental) circumstances. ${ }^{2}$ Although this view causes unrest among physicists, the scientific society in general takes for granted the definition given by dictionaries as the phenomenon where electromagnetic radiation and particles can exhibit either wave - like or particle - like behavior, but not both. ${ }^{3}$ One physicist Compton, concurring with Einstein, notes that these problematic experimental results could be easily explained by adapting the corpuscular view of the radiation. They are several experiments which demonstrate the corpuscular or undulatory behaviour of light and matter. For instance the photoelectric effect; the Compton effect; Grangier, Roger and Aspect correlation experiment.

Quantum mechanics gives rise to many paradoxes, which are sources of debates. Even physicists did not readily accept the duality concept. ${ }^{4}$ The photoelectric effect, usually referenced to support the particle - like nature of light in textbooks, simply prompted physicists' debates on the experimental evidence of the duality. ${ }^{5}$ For instance among pioneers of quantum theory, Bohr doubted the appropriateness of the duality concept even after the discovery of the Compton effect. The two concepts particle and wave, being mutually exclusive, interference phenomena made physicists wonder how the quantization concept could be in harmony with interference. At macroscopic level matter has entirely corpuscular properties, but Davisson - Germer experimental results showed that at microscopic level matter exhibits undulatory behaviour and is able to interfere. This revealed that matter is both particles and waves, effectively eliminating the old wave - or - particle dillemma with regard to light. ${ }^{2}$

Responding to the philosophical problem of wave-particle duality, Niels Bohr presented the complementarity principle, in concurrence with the uncertainty principle advanced by Werner Heisenberg 1927, which expresses that many - worlds interpretation, and the Seoul interpretation have been made. ${ }^{6-8}$ Variations in interpretations of the quantum theory result in different meanings, hence lack of consensus. For instance, the nature of the answer to the question of "What really happens during the two slit experiment?" could be different in accordance with one's preference for the interpretation of quantum theory. ${ }^{1}$ Entanglement and measurement are some of the problems which dog interpretation of issues on debates about the quantum theory. The distinctive characteristic of an entangled system is that a measurement on one part of the system can make an immediate influence on the other part of the system..$^{910}$ This is consistent with Heinsberg's principle of uncertainty. For instance, an electron cannot be said to have definitely passed through both slits, since the link 
between real behaviour of an electron and wave function is not clear In addition, consistent with the uncertainty principle, it can be validly argued that any attempt to figure out which slit electrons pass through will destroy the interference pattern in the experiment.

One basic question related to quantum mechanics which can be examined is, "What is the physical nature of a particle?" According to the wave - particle duality, particles have both properties of wave and corpuscle. In standard textbooks, this duality is often explained as the particle itself being a pointed object, with a wave like distribution, which is called the Copenhagen interpretation. However, there is no consensus on this view, as some physicists like Schrödinger, de Broglie and Einstein expressed reservations on such interpretation. ${ }^{11}$ Another crucial question that needs clarity is, "How can one explain the quantum behaviour of a free particle?" For instance is it possible for single electron to pass through two slits simultaneously as a light wave would do? Also, how can a single electron be diffracted from a crystal following the Bragg's diffraction law? In an attempt to find appropriate responses to these questions, Chang ${ }^{12}$ hypothesized that a sub - atomic particle is an excitation of the vacuum. This implies in the microscopic view, a particle is an excitation wave, while in the macroscopic view, it is a pointed object.

The questions now are that: Is this matter wave a physical wave? Is it carried by a medium? And, if yes, what is this medium?. ${ }^{11}$ The Copenhagen interpretation assumes a particle to be a pointed object that is like a tiny bullet, with a wave - like distribution. ${ }^{11,13}$ The wave function has statistical meaning, which focuses on the probability of a particle being at a specific location and time. In other words the wave function gives the probability of detecting the particle in the experiment. However, Chang ${ }^{12}$ believes a matter wave is a physical wave and a particle wave is a packet instead of a point - like object due to the following reasons. First, the elementary particle of light, the photon is a wave. Based on Maxwell's theory the photon is an oscillation of the electromagnetic field, hence it is a physical wave. In addition, for visible light the wavelength is about $400-700 \mathrm{~nm}$, implying the wave packet making up the photon spans over many wavelengths, clearly showing that the size of a photon cannot be regarded as point - like. Second, just like the photon, there is vast evidence showing the wave nature of an electron. ${ }^{11}$ For instance, establishment of Bragg's Law is hinged on diffraction of electrons by a crystal. ${ }^{14}$ If an electron was point - like it would bounce from one atom in the crystal, hence would not form an interference pattern. In addition, similar to light an interference pattern shown experimentally, by passing a single electron through a double slit shows that an electron is not point - like. ${ }^{15}$ Dirac theory can be used to explain the creation or annihilation of fermions, but cannot explain why bosons can also be created or annihilated, since they do not obey Pauli's exclusion principle. In this case Dirac theory cannot be used to explain hole creation in the negative energy states. Therefore, conventional quantum theories based on particle view have challenges in explaining many experimental facts.

\section{Levels of meaning of wave - particle duality}

Consensus concerning duality of microscopic objects can be arrived at by interpreting experimental results such as the photoelectric effect, Compton effect, or the two - slit experiment. Duality is not related to other core concepts of quantum theory such as wave function and its probabilistic interpretation. Also there is no relationship with formalism in quantum theory and interpretation concerning formalisn. ${ }^{16}$ Einstein - de Brogile relation plays a critical role in drawing meaning from related experiments. Cheong ${ }^{1}$ and Song ${ }^{1}$ suggest three levels of interpreting the wave - particle duality. At the first level, the core ideas of the quantum theory such as wave function, Born's probabilistic interpretation, Schro"dinger's equation, superposition principle, and the uncertainty principle, can be used to elaborate the meaning of wave - particle duality. At the second level consensus about some usefulness of theoretical ideas of the quantum theory is exhibited by success in their applications in making predictions of duality phenomena. However, as the third level, Cheong ${ }^{1}$ note that various interpretations provide different claims for the meaning and nature of duality. Diverse and mutually conflicting interpretations about the real behavior of microscopic objects, the real nature of the measurement process, and the status and role of quantum theory are suggested. The third level of the meaning of wave - particle duality encompasses these fundamental claims on the nature of duality, role and status of quantum theory. The third level meaning of divergence of duality related to various interpretations of quantum theory can be best illustrated through the two - slit experiment of electrons. Since the position and momentum of an electron are not predetermined before measurement, according to the Copenhagen interpretation, it is meaningless to ask about which slit an individual electron passed through in the two - slit experiment. However in contrast, Bohmian mechanics claims that an electron passes through one of the two slits in the experiment.

Based on one's philosophical position the purpose of interpreting the quantum theory could be predicting or explaining phenomena or making a reality claim in a hidden structure or the process of nature. ${ }^{1}$ A question which can be asked is: What is the hidden phenomena regarding the transitions between classical mechanics and quantum mechanics, specifically transitions between the particle nature and wave nature of matter? The premise for one's choice of interpretation determines the nature of answer to debates on issues pertaining to the quantum theory, as witnessed by divergence of views in debates on the wave - particle duality (Cheong and Song, 2014).

\section{Wave only, particle only or both?}

The challenging period for classical physics is illustrated by interference, diffraction and polarisation phenomena, which could only be explained by assuming that light has a wave nature. However, phenomena like black body radiation, the photoelectric effect and Compton scattering, defy the wave concept of electromagnetic radiation. Experimental results in 1923 by Compton an American physicist strongly confirmed the corpuscular nature of light. These results were also confirmed independently by Debye. ${ }^{17}$ It was observed that for an electromagnetic radiation incident on electrons of a light element, the scattered radiation was found to have a smaller frequency than the original frequency. This phenomenon is called the Compton Effect. This experiment confirmed both the law of conservation of energy, previously verified by the photoelectric effect, and the law of conservation of linear momentum. ${ }^{17}$ Thus, light has a dual nature, sometimes exhibiting the behaviour of waves and at other times showing the characteristics of particles. In 1923, a French PhD scholar, de Broglie (pronounced de Broy), extended the idea of the duality of light to the duality of matter.

According to Davisson and Germer ${ }^{18}$ Bohm built on de Brogile ideas postulating that every material particle is accompanied by a field which guides the motion of the particle. Evolving consistent with Schrödinger equation of quantum mechanics, this field called the pilot wave by de Brogile, and quantum field by Bohm ${ }^{19}$ is responsible for the undulatory behaviour of matter. ${ }^{2}$ Some eminent physicists of the old days found it difficult to come to terms with the statistical interpretation of the wave function and Heisenberg's principle of uncertainty. However, Bohr's analysed various new 
physics concepts which led to new trends in scientific attitudes and philosophical consequences specifically the wave and particle aspects of matter which are opposing but complementary, consistent with the principle of complementarity. As expressed by Niels Bohr ${ }^{20}$ the complementarity principle holds that objects have certain pairs of complementary properties which cannot all be observed or measured simultaneously. Some complementary properties Bohr considered are:

\section{Position and momentum;}

\section{Spin on different axes;}

III. Wave and particle - related properties;

IV. Value of a field and its change (at a certain position); and

\section{Entanglement and coherence. ${ }^{21}$}

Microscopic systems are neither pure particles nor pure waves, they are both. The particle and wave manifestations do not contradict or preclude one another, but, as suggested by Bohr, they are just complementary. Both concepts are complementary in describing the true nature of microscopic systems. Any quantum system is neither pure particle nor pure wave, but depends on equipment used to detect, implying all particles have capacity to display either "particle" or "wave" features. For instance in the double - slit experiment if one slit is blocked the particle aspect will be observed, and the wave aspect observed when both slits are open. Therefore particle and wave features are embedded in the electron (all materials) and by modifying conditions and detection tools, one aspect of the electron can be supressed, while the other is kept. Three elements of de Brogile's Theory Lima et al..$^{22}$ present are first the theory refers to every being in the world; second, it is a symmetrically dual theory, implying that there are no particles that behave as matter, and waves that behave as particles. This implies all beings are composed of both particles and oscillatory phenomena; and third de Brogile's theory is relativistic. In other words, though both the particle and wave behaviours are embedded in matter, they cannot be simultaneously exhibited. Emphasis put on one wave - particle attribute during measurement, is done at the expense of the other. For instance, exhibiting particle properties of matter in an experiment, does not give any information on the wave aspect and vice versa. ${ }^{17}$ Illustrating this view Saleem ${ }^{17}$ notes that cloud chamber tracks illustrate the particle nature of matter, but not the wave nature. Similarly, interference and diffraction experiments show the wave nature of light but the particle nature. Therefore without the complementary principle, quantum mechanics cannot produce accurate results.

According to Saleem ${ }^{17}$ all possibly available knowledge about the characteristics of a microscopic entity, like an electron, is contained in the wave function. When under certain conditions a microscopic entity shows properties which at macroscopic level are attributed to particles, it said to behave as a particle. If the same microscopic entity under different conditions exhibits characteristics which are assigned to waves at macroscopic level, then the entity is said to be behaving as a wave. It is therefore clear that the behaviour of a microscopic entity is determined by the same wave function, depending on prevailing conditions. Particles and waves are mutually exclusive in classical physics, since they exhibit different behaviours. Electrons, protons and any other microscopic particles behave neither as classical particles nor classical waves. When we subject an electron to Compton scattering, we observe only its particle aspects, but when we involve it in a diffraction experiment (double - slit experiment), we observe its wave behaviour only. So if we measure the particle properties of a quantum system, this will destroy its wave properties and vice versa, consistent with Heisenberg's uncertainty principle . Any measurement gives either one property or the other, but never both at once. We can get either the wave property or the particle but not both of them together.

\section{Matter waves: criticisms of de brogile equation}

The conceptual breakthrough of presenting the idea that all matter had a wave like nature, by de Brogile in his doctoral thesis in 1924, was confirmed by the electron diffraction experiment by Lester Germer first, and by Clinton Davisson three years later. In addition Thomson, obtained interference patterns with electrons thereby again confirming the wave behaviour of electrons which paved way for further development of quantum mechanics in the late $20 \mathrm{~s}$ and the $30 \mathrm{~s}^{2}$ De Brogile suggested that that all material particles should display a dual wave - particle behaviour (the wave - particle duality present in the light must also occur in matter). Starting from the momentum of a photon $\mathrm{p}=\mathrm{hv} / \mathrm{c} \quad=\mathrm{h} / \lambda$, can be generalized to any material particle with non - zero rest mass. Put in strict but simple mathematical form, the de Brogile relation assigned every physical particle (like an electron) a wavelength and a frequency. ${ }^{23}$

Clarity which lacks is the meaning of relating wavelength and frequency to a physical object. While this state of affairs prevails, the hypothesis by de Brogile that matter in motion could be perceived as a wave was confirmed by Davisson and Germer ${ }^{18}$ and, independently, by Thomson and Reid. ${ }^{14}$ Now it is not known whether the analogous diffraction phenomena occur with other elementary particles like neutrons and protons, or even with much larger atoms and molecules. In matter diffraction experiments for now continuous flow of particles has been considered, and the nagging question is whether the diffraction pattern could be explained in terms of some collective behaviour of these particles instead of employing de Broglie waves. ${ }^{2}$ This is an area for research to ascertain. Some physicists present the view that macroscopic objects such as electrons have too small wavelengths such that their wave behaviour cannot be observed or be detected. The general rule is given as whenever the de Broglie wavelength of an object is in the range of or exceeds its size, the wave nature of the object is detectable. However, if its de Broglie wavelength is too small compared to its size, the wave behaviour of this object is undetectable The question to ask is: Is this real or it is an attempt to make an extrapolation for big particle behaviour from the behaviour of microscopic particles, so that the de Brogile equation remains valid at macroscopic level? There is need for experimental evidence to validate this.

Many puzzles exist about concepts in quantum mechanics, although it has been a great successful theory in physics for over one hundred years. Contrary to classical physics wave equations based on well - established physical laws, conjectures have been the basis of quantum wave equations derivation, the rationale being that these equations can lead to results consistent with experiments. ${ }^{13,24}$ However, basing quantum wave derivation on conjecture is totally not satisfactory, since it is at variance with the tradition of physics of always wanting to know the physical basis behind a theory. This is a criticism de Brogile equation faces, that it is a product of conjectures rather than experimental work. In this vein effort should be put by physicists to measure the quantal matter wave, hence providing experimental evidence, to support such claims. With knowledge like the Schrödinger, Dirac and de Brogile equations we think we have a good understanding of the structure of an atom, but what about the detailed structure in time and space of the state of transition and the resulting photon? When a theory successfully predicts various phenomena, it does not follow that it reveals the hidden structure of 
nature in a literal sense. Also an observation regarded as a fact may be considered wrong due to the theory ladenness of the observation. ${ }^{25}$ This is another challenge faced by de Brogile equation.

\section{Conclusion}

Particles and waves are mutually exclusive in classical physics, since they exhibit different behaviours. In quantum mechanics, as postulated by de Brogile both the particle and wave behaviours are embedded in matter, but they cannot be simultaneously be exhibited. While de Brogile is applauded for a break through which provided knowledge for guiding further development of quantum mechanics, there are also criticisms which need attention, and these are:

I. de Brogile equation derivation is a product of conjectures rather than experimental work. To date no quantal matter wave has been measured.

II. Lack of clarity on the meaning of relating wavelength and frequency to a physical object in the de Brogile equation.

III. Transitions between classical mechanics and quantum mechanics are not clear in de Brogile equation.

Premised on the foregoing discussion, complexity of interpretation of the wave - particle duality is a fact, which indicates that debates related to interpretation of the quantum theory, and specifically de Brogile equation, will not end in a foreseeable future. Therefore physicists should continue debating these issues as well as putting effort to provide experimental evidence, to substantiate claims.

\section{Acknowledgments}

None.

\section{Conflicts of interest}

The author declares there is no conflict of interest.

\section{References}

1. Cheong WY, Song J. Different Levels of the Meaning of Wave-Particle Duality and a Suspensive Perspective on the Interpretation of Quantum Theory. Sci \& Educ. 2014;23:1011-1030.

2. Jagielski B. Master of Science in Physics Thesis submitted to the University of Oslo. 2009.

3. Gray JH, Alan I. Dictionary of Physics. Longman. 1990.

4. Mehra J, Rechenberg H. The quantum theory of Planck, Einstein, Bohr, and Sommerfeld: Its foundation and the rise of its difficulties 19001925. New York: Springer. 1982. p. 511-532.

5. Klassen S. The photoelectric effect: Reconstructing the story for the physics classroom. Science \& Education. 2010;20(7- 8):719-731.
6. Bohr N. Can quantum-mechanical description of physical reality be considered complete? Physical Review. 1935;48:696-702.

7. Bohm D. A suggested interpretation of the quantum theory in terms of "hidden" variables, I and II. Physical Review. 1952;85:166-193.

8. Zhang HI. Epistemic subject and epistemological structure of science. Korean Journal for Philosophy of Science. 1998;1(1):1-33.

9. Zbinden H, Brendel J, Tittel W, et al. Experimental test of relativistic quantum state collapse with moving reference frames. Journal of Physics A. 2011;34:7103-7111.

10. Dickson M. Non-relativistic quantum mechanics. In J Butterfield et al., editors. Philosophy of Physics Part A. Amsterdam: Elsevier. 2007.

11. Messiah A. Quantum Mechanics. Vol. 1. New York: John Wiley \& Sons. 1965.

12. Chang $\mathrm{CD}$. On the wave nature of matter: A transition from classical mechanics to quantum mechanics. 2017.

13. Slater CJ. Concepts and Development of Quantum Physics. Dover Publications. 1969.

14. Thomson PG, Reid A. Diffraction of Cathode Rays by a thin Film. Nature. 1927;119:890.

15. Bach. Controlled double-slit electron diffraction. New J Phys. 2013;15:033018.

16. Cushing JT. Philosophical concepts in physics: The historical relation between philosophy and scientific theories (Chap. 23). New York: Cambridge University Press. 1998.

17. Saleem M. The failure of classical physics and the advent of quantum mechanics. IOP Pulishing Ltd. 2015.

18. Davission JC, Germer HL. The scattering of electrons by a single crystal of nickel. Nature. 1927;119:558-560.

19. Bohm D, Hiley BJ. The Undivided Universe: An ontological interpretation of quantum theory. Routledge. 1995. p. 416.

20. Harris EW. The Physics of Consciousness. Cambridge, Massachusetts: Perseus. 2010

21. Cramer JG, Herbert N. An Inquiry into the Possibility of Nonlocal Quantum Communication (Report). 2015.

22. Lima WN, Nascimento MM, Ostermann F, et al. Louis de Broglie's wave-particle duality: from textbooks'blackboxes to a chain of reference presentation. 2020.

23. de Broglie L. Recherches sur la théorie des quanta, doctoral thesis. Paris, 1924; as translated by AF Kracklauer. 1924.

24. Dirac MAP. The Principles of Quantum Mechanics. Oxford: Clarendon Press. 1981.

25. Hanson NR. Patterns of discovery: An inquiry into the conceptual foundations of science (Chap.1). Cambridge University Press, London. 1961. 